\title{
Harina de forrajes en la alimentación animal
}

\author{
Fodder flour in animal feed \\ Gutiérrez Castro Litsy ${ }^{1}$ y Güechá Castillo Andrea ${ }^{2}$ \\ ${ }^{1}$ Médico Veterinario Zootecnista, (c)MSc, Universidad de los Llanos y \\ ${ }^{2}$ Médico Veterinario Zootecnista, Universidad de los Llanos \\ litsy.gutierrez@unillanos.edu.co
}

Recibido 10 de Mayo 2016, Aceptado 29 de Octubre 2016

\section{RESUMEN}

En este documento se destaca la importancia de las especies arbóreas empleadas en la alimentación de animales de interés zootécnico, y cómo las diferentes alternativas de conservación permiten suministrar un alimento de excelente calidad nutricional en periodo de escasez. Como es sabido, la alimentación eficiente es uno de los aspectos más importantes en la producción animal, puesto que de ésta depende no solo el rendimiento zootécnico de los animales, sino también la rentabilidad económica del productor. Para brindar una buena dieta, se deben suministrar alimentos con la cantidad necesaria de nutrientes en las condiciones físicas óptimas para qué los mismos sean utilizados metabólicamente de forma eficiente, y así poder obtener el máximo rendimiento en la producción. El suministro del alimento en forma no convencional permite explorar nuevas alternativas que brinden alimentos de buena calidad. En la búsqueda de fuentes alimenticias de bajo costo en el trópico, se ha incluido la evaluación de follajes arbóreos por su alta disponibilidad; la utilización de harinas de follajes de plantas se ha considerado como opción para la sustitución parcial o total de proteína proveniente principalmente de granos y cereales para la alimentación animal, siendo en la actualidad una de las estrategias de mayor investigación, en donde al facilitar su inclusión en las dietas balanceadas de los animales, se convierte en una alternativa económica que permitirá sustituir en parte el uso de alimento concentrado comercial. Especies arbóreas como el matarratón (Gliricidia sepium), leucaena (Leucaena leucocephala), guacimo (Guazuma ulmifolia), cayeno 
(Hibiscus rosa-sinensis) y morera (Morus alba) son de interés para el productor debido a su potencial de producción (21.93-30.6 ton/ha/MS).

Palabras clave: Suplemento, dieta, nutrientes, leguminosas, arbóreas.

\begin{abstract}
In this document highlights the importance of the tree species used in the feeding of animals of zootechnical interest, and how the different conservation alternatives allow to supply a food of excellent nutritional quality in times of scarcity. As it is known, the efficient feeding Is one of the most important aspects in animal production, because of this depends not only on the zootechnical performance of the animals, if not also the economic profitability of the producer. To provide a good diet, the food must be supplied with the necessary amount of nutrients in optimal physical conditions so that the same be metabolically used of form efficient and thus to obtain the maximum yield in the production. The food supply in nonconventional form allows to explore new alternatives which provide good quality food in the search for low-cost food sources in the tropics, the evaluation of tree foliage has been included due to its high availability; the use of flours of plant foliage has been considered as an option for the partial or total substitution of protein mainly from grains and cereals for animal feed being currently one of the strategies of greater research, where facilitating their inclusion in the balanced diets of animals becomes an economic alternative which will replace in part the use of commercial concentrated food. Tree species such as Gliricidia sepium, Leucaena leucocephala, Guazuma ulmifolia, Hibiscus rosa-sinensis and Morus alba are of interest to the producer because of their production potential (21.9330.6 ton/ha/DM).
\end{abstract}

Keywords: Supplement, diet, nutrients, legumes, arboreal.

\title{
RESUMO
}

Em este documento destaca a importancia das espécies arbóreas usadas na alimentação de animais de interesse zootécnico, e como as diferentes alternativas 
de conservação permitem gerenciar um alimento de excelente qualidade nutricional em tempos de escassez. Como é sabido, a alimentação eficiente é um dos aspectos mais importantes na produção animal, sendo que desta depende não só do desempenho zootécnico dos animais, senão também a rentabilidade econômica do produtor. Para proporcionar uma boa dieta, devem ser suminitrados alimentos com a quantidade de nutrientes necessários em condições físicas superiores de modo a que eles são utilizados metabólicamente de forma eficiente, e assim poder obter o máximo desempenho na produção. O fornecimento de alimentos de forma não convencional permite explorar novas alternativas que proporciona alimentos de boa qualidade na busca de fontes de alimentos de baixo custo nos trópicos, foi incluído a avaliação da folhagem da árvore por seu alta disponibilidade; o uso de farinha de folhagem das plantas tem sido considerado como uma opção para a substituição parcial ou total da proteína principalmente a partir de grãos e cereais para a alimentação animal sendo atualmente uma das estratégias de maior investigação, onde para facilitar a inclusão nas dietas equilibradas de animais se torna em uma alternativa económica que vai substituir parcialmente a utilização de ração comercial. Espécies de árvores como Gliricidia sepium, Leucaena leucocephala, Guazuma ulmifolia, Hibiscus rosa-sinensis e Morus alba são de interesse para o produtor devido ao seu potencial de produção (21.93-30.6 ton/ha/MS).

Palavras-chave: Suplemento, dieta, nutrientes, leguminosas, arbóreo.

\section{INTRODUCCIÓN}

El continuo crecimiento de la población y el deterioro de los recursos naturales que sustentan la producción de alimentos justifican la investigación en opciones más adecuadas, en donde se le brinde la importancia a algunos follajes para que sean valorados como opción para la suplementación nutricional total o parcial en la dieta animal. Actualmente en el trópico, se pretende establecer un complemento nutricional con el uso de productos proteicos y energéticos no tradicionales utilizados en nutrición animal, forjando la búsqueda e implementación del uso de nuevas materias primas que no compitan con la alimentación humana y mucho 
menos dependan de la importación de granos y cereales, que origina el elevado precio de la alimentación y por ende se ve reflejado en los altos costos en un sistema productivo (Sarria et al., 2005); a esto se le suma la falta de tecnificación que afrontan los sistemas de producción pecuaria que tienen dificultades en la cantidad, calidad y provisión de alimentos (Estupiñan et al., 2009).

Por otro lado, en los últimos años en países tropicales ha cobrado gran importancia la introducción de follajes de árboles y arbustos en la alimentación de rumiantes y no rumiantes basada en la disponibilidad local y composición nutricional, sin embargo la composición bromatológica no refleja el valor nutritivo potencial de estas fuentes, que depende entre otros factores, de la estructura química, calidad de la fracción fibrosa y presencia de factores antinutricionales. Esto permite predecir sus efectos en las funciones gastrointestinales y metabólicas del organismo que contribuye a lograr formulaciones óptimas en la ración que se reflejen con un mejor comportamiento productivo (Savon et al., 2005).

Las principales investigaciones realizadas en los últimos años se han enfocado en los altos contenidos proteicos de las plantas, de tal forma que las harinas suelen presentarse como una opción positiva con el fin de transformar el follaje y preservar su calidad nutricional para que pueda ser empleada como suplemento proteico, ya sea combinada con otras materias primas o sustituyendo de forma parcial los concentrados (Savon et al., 2005).

\section{CONSERVACIÓN DE FORRAJES}

Los forrajes son la base de alimentación de los rumiantes, cuando estos tienen un uso adecuado permiten satisfacer completamente las necesidades nutricionales de los animales. Entre las familias más importantes se encuentran las gramíneas y leguminosas en donde la calidad nutritiva depende del tiempo de maduración de las plantas, y los componentes más importantes a destacar en los forrajes son la proteína, fibra bruta y minerales (Parsi et al., 2001).

Pero antes de decidir cultivar o mantener una pradera, se debe tener en cuenta ciertos factores como reconocer que especies forrajeras son nativas de la región, y 
su uso y calidad nutricional, esto con el fin de determinar si el forraje elegido cumple con los requerimientos para la especie a alimentar, debido a que es primordial reconocer el porcentaje de proteína que aporta en la dieta e identificar si es necesario implementar un cultivo mixto con leguminosas $u$ otras especies que permitan complementar el aporte de proteína (Moreno y Molina, 2007).

La producción de forrajes verdes es estacional, y para lograr un óptimo aprovechamiento de los excedentes de biomasa (forraje y/o follajes) producidos en algunas épocas del año, hay que conservar el material verde en las mejores condiciones posibles, de manera que permita su utilización a largo plazo sin perder su valor nutricional. Para esto, existen varias técnicas y métodos de conservación; los más empleados son: el ensilaje, a base de forraje fresco o procesado y el henolaje (Parsi et al., 2001).

\section{Ensilaje}

El ensilaje es la fermentación de los carbohidratos solubles del forraje por medio de bacterias epifíticas que producen ácido láctico y en menor cantidad ácido acético, en donde el material ensilado disminuye el $\mathrm{pH}$ evitando la presencia y crecimiento de microorganismos que alteren su conservación. Existen diferentes técnicas para elaborar el ensilaje (Wagner et al., 2013), algunos de ellos son:

1. Silo bolsa: es el más empleado por los pequeños productores, este silo se realiza de forma manual, en el cual se emplean bolsas de un espesor de 2 $\mathrm{mm}$, y se debe almacenar sobre una superficie lisa y protegerlas con el fin de evitar que se rasguen las bolsas y perder el proceso realizado (Figura 1).

2. Silo trinchera: Se construye bajo el nivel del suelo en terrenos de relieve inclinado, pero pueden presentar pérdidas adicionales por filtración de humedad.

3. Silos bunker: Se construyen sobre el nivel del suelo, las paredes y piso pueden ser de concreto, lamina o madera.

4. Silos de montón: También silo de pila, en este silo se acumula el forraje picado y se tapa: es un silo muy económico pero presenta altos porcentajes de 
pérdidas. Se deben construir en sitios de piso firme, además de incluir en sus costos la adquisición de un plástico calibre 7 u 8 para proteger el silo.

5. Silos en canecas y tanques: Se utilizan canecas plásticas con capacidad para 200 litros y tanques de 500 y 1000 litros., son económicos y facilita el llenado y apisonado del forraje.

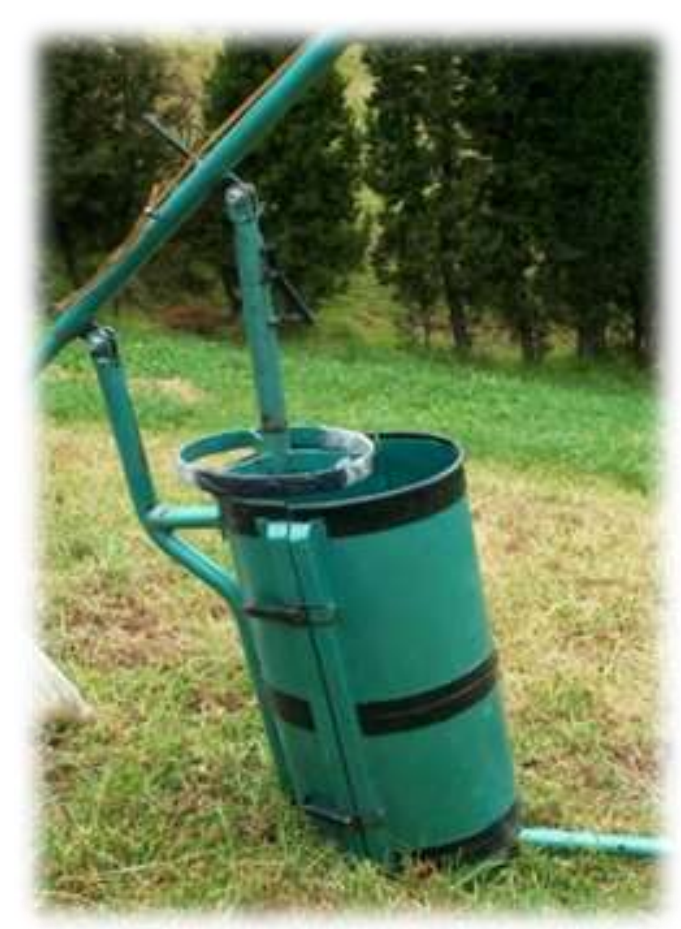

Figura 1. Ensilaje manual

Fuente: Fotografía tomada por Andrea Güechá, (2016)

Este proceso de ensilaje, es empleado para almacenar alimento y ofrecerlo en tiempos de escases con el fin de conservar su calidad y palatabilidad durante el tiempo (Garcés et al., 2004). Algunos de los factores que se deben tener en cuenta para llevar a cabo un buen ensilado, son: conocer qué tipo de cultivo se desea ensilar (cereales, gramíneas o leguminosas) ya que la altura de corte, el tamaño de partículas, nivel de humedad, resistencia a compactación, calidad y velocidad de fermentación, influyen en el éxito de este proceso (Figura 2) (Paziani, 2004; Wagner et al., 2013). 


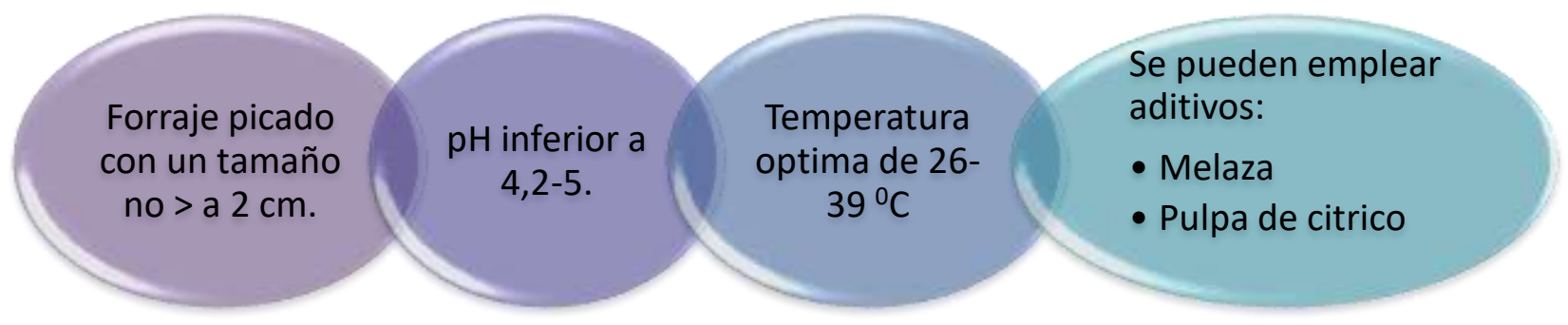

Figura 2.Factores que se deben tener en cuenta para un buen ensilaje. Fuente: Adaptado de Garcés et al., (2004) y Paziani (2004)

\section{Henificación}

Es un proceso por el cual los forrajes son deshidratados en forma natural (secado al sol) o de forma mecánica (en horno secador) por un tiempo de 24 a 72 horas con el fin de conservar el cultivo o forraje en su máxima calidad nutricional para que puede ser almacenado y usado posteriormente para el consumo animal, especialmente en momentos de escases de alimento (Blass et al., 2011). Para producir un heno de buena calidad se debe tener en cuenta algunos aspectos como el estado fisiológico del forraje, condiciones climáticas durante la cosecha y otros factores que se resaltan en la Figura 3.

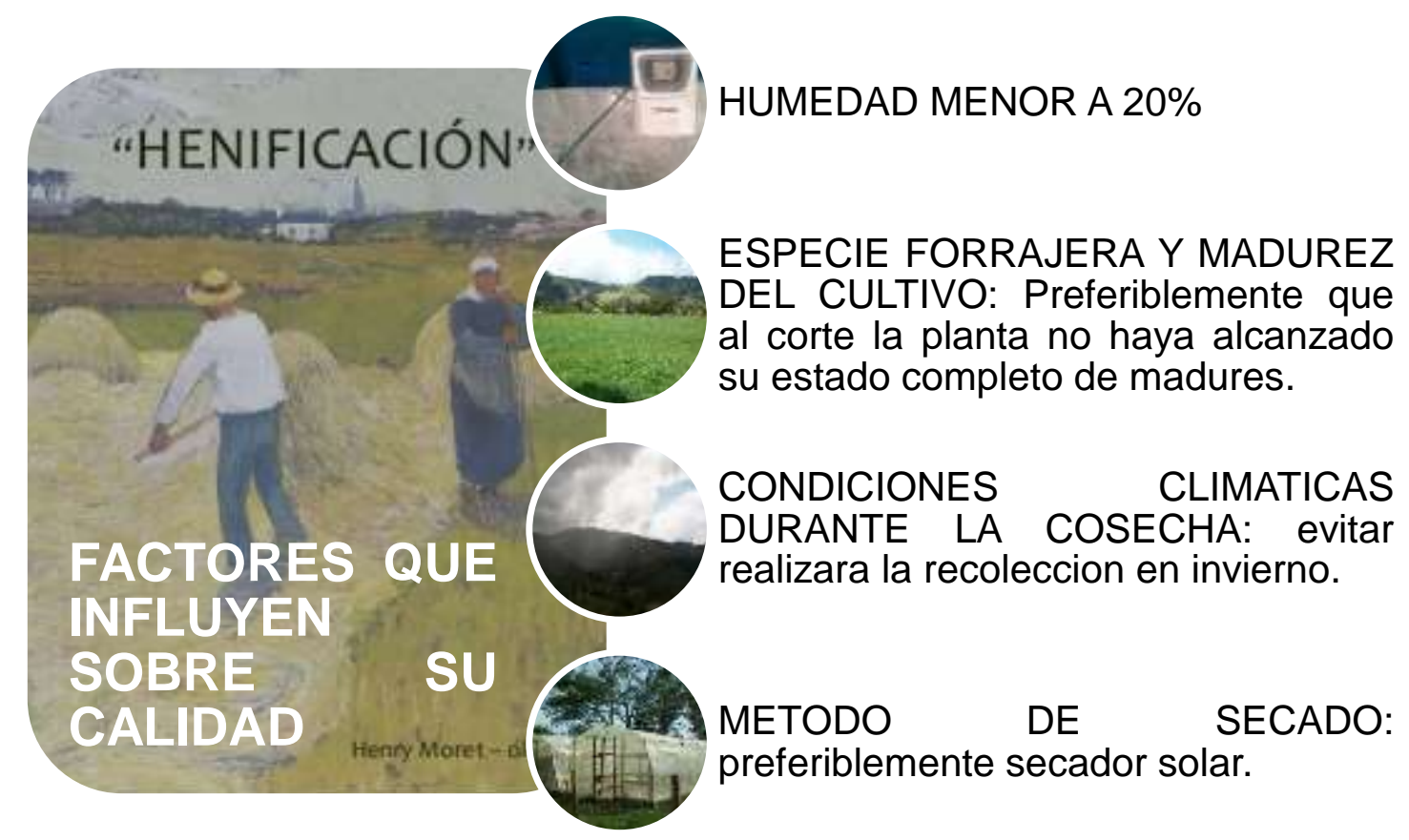

Figura 3. Factores que influyen sobre la calidad de henificación.

Fuente: Tomado de Parsi et al., (2001). Fotografía principal tomada por Henry Moret, fotografías secundarias tomadas por Andrea Güechá, (2016) 


\section{Harinas}

La transformación de forrajes en harina se ha convertido en una actividad creciente de implementación especialmente en zonas del trópico con variabilidad climática que presentan periodos críticos extremos, donde no se logra suplir las necesidades alimenticias de los animales (Mejía et al., 2013). Los forrajes de hojas grandes son los más utilizados en harinas por la diversidad de plantas que se encuentran en la región (Figura 4), además de su alta producción de biomasa que lo hace atractivo al productor. La ventaja de deshidratar el forraje permite minimizar las pérdidas nutricionales del forraje durante la transformación del producto, asimismo el proceso de secado debe ser homogéneo y controlado con el fin de disminuir algún tipo de contaminación (Sarria et al., 2009).

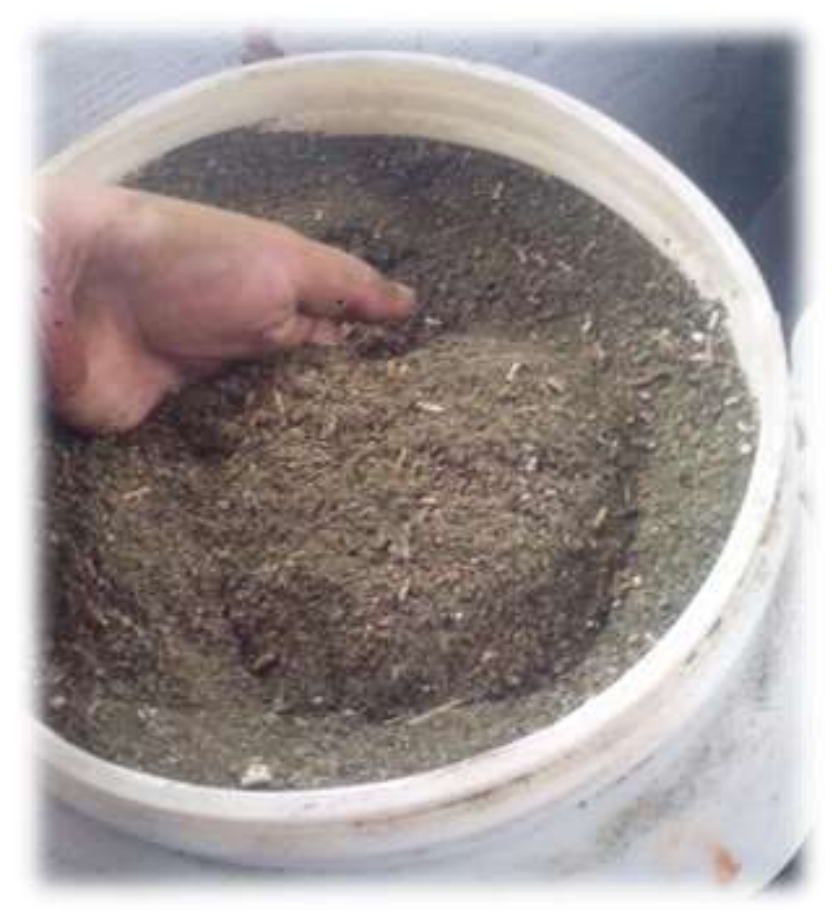

Figura 4. Harina Tithonia diversifolia.

Fuente: Fotografía tomada por Andrea Güechá, (2016)

La ventaja que tiene la conservación en forma deshidratada radica en que una vez transformados en harinas, pueden ser incluidas en una gran diversidad de suplementos, debido a su textura, además de permitir su almacenamiento por largos períodos de tiempo sin afectar su valor nutritivo. Asimismo mediante el empleo de la tecnología de fabricación de harina, se puede ayudar a mejorar el 
consumo de la biomasa producida por algunas arbóreas que son poco palatables en estado fresco como por ejemplo Gliricidia sepium y Leucaena leucocephala (Savon et al., 2005).

\section{RESULTADOS SOBRE EL USO DE HARINAS DE FOLLAJE EN ANIMALES}

\section{Implementación en rumiantes}

Algunos estudios realizados con árboles y arbustos, integrándolos a sistemas de producción con rumiantes, han permitido evaluar la variedad de forrajes que actualmente no tienen ningún uso y ampliar la utilidad de aquéllas que normalmente tienen otros propósitos. Especies arbóreas como el matarratón (Gliricidia sepium), leucaena (Leucaena leucocephala), guacimo (Guazuma ulmifolia), cayeno (Hibiscus rosa-sinensis) y morera (Morus alba) son de interés, y su importancia radica en su contenido de proteína (14-26\%), grado de digestibilidad (superior al 60\%) y potencial de producción 21.93 y 30.6 ton/ha/MS) comestible para morera y cayeno, respectivamente, lo cual logra que estos forrajes se asemejen a un concentrado en términos de contenido nutricional, despertando el interés de utilizarlos como suplementos en regiones tropicales (Meza et al., 2014).

Un estudio realizado por Mata et al., (2006) para conocer el efecto de suplementación con harina de tres arbustivas forrajeras tropicales (matarratón, morera y cayeno), en el comportamiento productivo y fermentación ruminal de corderos en crecimiento, estimó que la harina de cayeno $(\mathrm{HC})$ mostró una respuesta similar a la del concentrado comercial (CC), en relación al consumo $(181.6 \mathrm{~g}$ y $167.2 \mathrm{~g})$ y la ganancia de peso diaria $(81.6 \mathrm{~g}$ y $77.1 \mathrm{~g})$ y que la menor respuesta se obtuvo con el matarratón (HM) (97.7g y $48.1 \mathrm{~g})$, respectivamente, por lo cual se recomienda $\mathrm{HC}$ como sustituto de $\mathrm{CC}$ para corderos en pastoreo en el trópico. La harina de frutos de pijiguao o chontaduro (HIP) surge como un ingrediente no tradicional para la alimentación animal, y representa una excelente fuente energética rica en azúcares, grasa, almidón y ácidos grasos. Otro estudio realizado por estos mismos autores determina los diferentes componentes del 
Bactris gassipaes a partir de un análisis químico y mineral arrojando valores de proteína cruda de $6.58 \%$, grasa $12.58 \%$, cenizas $1.98 \%$, calcio $0.08 \%$, fosforo $0.11 \%$, magnesio $0.05 \%$, potasio $0.39 \%$ y 20.22 , 4 y 3 ppm para hierro, zinc, cobre y manganeso. El contenido de energía metabolizable estimada HIP fue de 2,964 Mcal $/ \mathrm{kg}$, los contenidos de fibra, expresados como FDN y FDA fueron de 21,16 y $10.44 \%$ respectivamente, degradabilidad in vitro de la materia seca de 80\%; por lo anterior, se concluyó que la HIP constituye una fuente energética alternativa para la alimentación de rumiantes por su bajo contenido de metabolitos secundarios como fenoles y taninos, y presentar altos valores de degradabilidad in vitro. Así mismo, Pizzani et al., (2008), manifiestan que los sistemas agrosilvopastoriles (Figura 5) en sus diferentes modalidades ofrecen de forma alternativa y sostenible recursos alimenticios con potencialidades nutricionales, e igualmente exponen que estos sistemas tienen su relevancia especialmente en el trópico, donde la necesidad de la ganadería por pasturas, produce una enorme presión en las áreas de bosques, puesto que la dieta base de los rumiantes (el forraje) aporta principalmente energía.

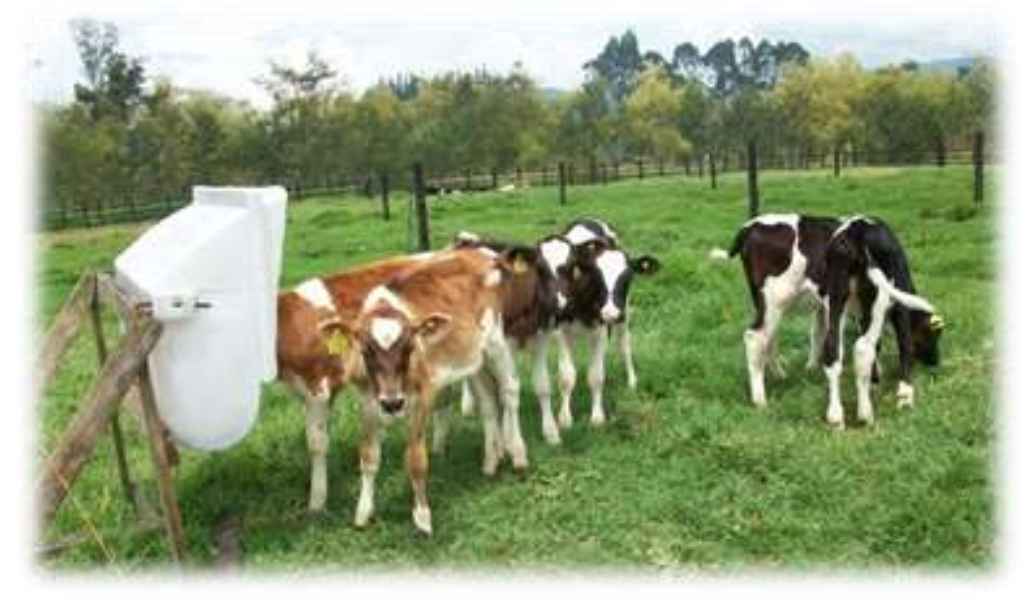

Figura 5. Suplementación en terneros.

Fuente: Foto tomada por Andrea Güechá (2016)

Cino et al., (2012) evaluaron la posibilidad económica del empleo de Tithonia diversifolia (TD) como harina de forraje en dietas integrales destinadas a terneros lactantes. Los tratamientos con porcentajes de inclusión de TD fueron: $5 \%, 10 \%$ y $15 \%$, en donde estos registraron los menores valores de costos/animal/día (2.11, 
2.01, 2.10 USD respectivamente), en comparación con el valor de la dieta control 2.12 USD; asimismo el uso de la harina como elemento de la dieta integral constató menor nivel en sus costos directos (costo/kg) con respecto a los registrados en la dieta control (1.70. 1.67, 1.66, $1.62 \mathrm{USD} / \mathrm{kg}$ respectivamente). Los resultados preliminares demuestran las posibilidades económicas de su manejo en terneros lactantes con una mayor utilización de recursos naturales.

\section{Implementación en no rumiantes}

El uso de harina de hojas de forrajes se ha incrementado en el trópico dentro de la alimentación de cerdos, Sarria et al., (2009) en un estudio determinó la composición de las hojas de la planta bore (Xanthosoma sagittifolium) y dos árboles: nacedero (Trichanthera gigantea) y morera (Morus alba), su valor nutritivo en cerdos jóvenes y la ingesta voluntaria en cerdas adultas. El contenido de proteína estuvo entre 170 y $240 \mathrm{~g} / \mathrm{kg}$ de materia seca (MS) y de fibra detergente neutra (FDN) entre 218 y $398 \mathrm{~g} / \mathrm{kg}$ MS. Las hojas son fuente de calcio (hasta 69 $\mathrm{g} / \mathrm{kg} \mathrm{MS}$ ), potasio, hierro y manganeso, y la ingesta alcanzó $3.4 \mathrm{~kg}$ de hojas frescas por día (0.51 kg MS) y 1.0-1-1 kg de harina de hojas por día. En este estudio se concluyó que la baja densidad energética (1674 y $2037 \mathrm{kcal} / \mathrm{kg}$ ) es el principal factor limitante de los follajes para la nutrición de cerdos jóvenes que requieren promedio de $3400 \mathrm{kcal} / \mathrm{kg}$; pero son buena fuente de minerales y de proteína balanceada, con opciones interesantes en cerdas adultas, por su mayor capacidad de consumo, la cual se duplica cuando se brinda en forma de harina en lugar de hojas frescas.

Trompiz et al., (2000) evaluaron el efecto de la sustitución parcial del alimento concentrado balanceado por harina de yuca (HFY) (0 y $15 \%)$ sobre el comportamiento productivo en cerdos durante la etapa de crecimiento, en donde los tratamientos fueron similares $(P<0.05)$ para ganancias diarias de peso (GDP) $(0.660$ y $0.665 \mathrm{~kg})$ y ganancia total de peso (GTP) $(27.7$ y $27.9 \mathrm{~kg})$ respectivamente, de igual forma la conversión alimenticia (CA) no se vio afectada por los tratamientos evaluados (4.34 y 4.95); por lo tanto, estos resultados 
permiten recomendar la HFY en un $15 \%$ de sustitución en la dieta durante la etapa de crecimiento en cerdos.

Estupiñan, (2009) en un estudio en cerdos de crecimiento donde el CC fue substituido hasta por $20 \%$ de harina de morera, se encontró que el mejor nivel de reemplazo fue el 15\%, incrementando las GDP hasta 740 g/día, en comparación con CC que fueron $680 \mathrm{~g}$, adicionalmente fue mayor la rentabilidad con este nivel de reemplazo; de igual forma se reporta que el consumo total de alimento (CTA) no registro diferencias para 0,6 y $12 \%$ (230, 232 y $235 \mathrm{~kg}$ respectivamente), al igual que la ganancia de peso $(62,59$ y $61 \mathrm{~kg})$. La CA fue diferente $(P<0.05)$, demostrando un efecto depresivo con el incremento de la harina de morera en inclusión de 12, 18 y 24\% (3.89, 4.14 y 4.68 kg respectivamente). González et al., (2006) sugieren que la harina de hojas de morera puede ser introducida hasta en un $20 \%$ en dietas para cerdos en crecimiento influyendo muy poco en la digestibilidad y comportamiento fisiológico del tracto digestivo.

En un ensayo con la finalidad de evaluar el efecto de raciones con diferentes niveles de HFY (harina de yuca): 0, 10 y $20 \%$ sobre el rendimiento productivo de cerdos en etapa de engorde, se reportó que los tratamientos fueron similares para GPD: $0.764,0.744$ y $0.699 \mathrm{~kg}$ y CTA $130.55,129.11$ y $130.68 \mathrm{~kg}$ respectivamente, indicando que la HFY constituye un recurso no tradicional, nativo y una excelente fuente proteica para ser utilizado en un $20 \%$ en la alimentación de cerdos en la etapa de engorde (Trompiz et al., 2002).

Cancio et al., (2010) exponen que la elevada producción de biomasa vegetal en el trópico y la existencia de numerosas especies con alto potencial alimenticio para animales herbívoros, incentiva el estudio de estrategias de alimentación que consideran el justo valor de los forrajes; además determinaron que el forraje de nacedero proporciona altos niveles de CA (6.75), GDP (32 g) y rentabilidad, en dietas para conejos de engorde donde se favorece con la inclusión hasta del 50\% del CC y el forraje a voluntad, sin mostrar efectos tóxicos que acarreen trastornos metabólicos o muertes de los gazapos. Por otra parte Meza et al., (2014) resaltan la alta disponibilidad de plantas arbustivas y arbóreos útiles para cuyes, en donde 
además sustentan la posibilidad de incluirlas en forma de harina en dietas balanceadas, por lo cual evaluaron el comportamiento productivo de cuyes (Figura 6) con la inclusión del $20 \%$ de harinas derivadas de follajes arbustivos y arbóreos tropicales, donde los mejores resultados $(\mathrm{P}<0.01)$ obtuvieron para $G T D$ en g/animal/d, CA y rendimiento en canal $(R C)$ en porcentaje, la registraron los tratamientos: T0 (dieta 100\% balanceada: $8.71,5.57$ y $76.30 \%$ ), T1(80\% dieta y $20 \%$ harina de morera: $8.80,5.04$ y 73.08$)$ y T3 (80\% dieta y $20 \%$ harina de botón de oro: $8.43,5.38$ y 77.67 ) respectivamente, concluyendo que el tratamiento T3 demostró una mejor eficiencia de los parámetros productivos y rentabilidad, y por lo tanto una reducción del costo de alimentación en $26 \%$ al utilizar dicho sistema de alimentación.

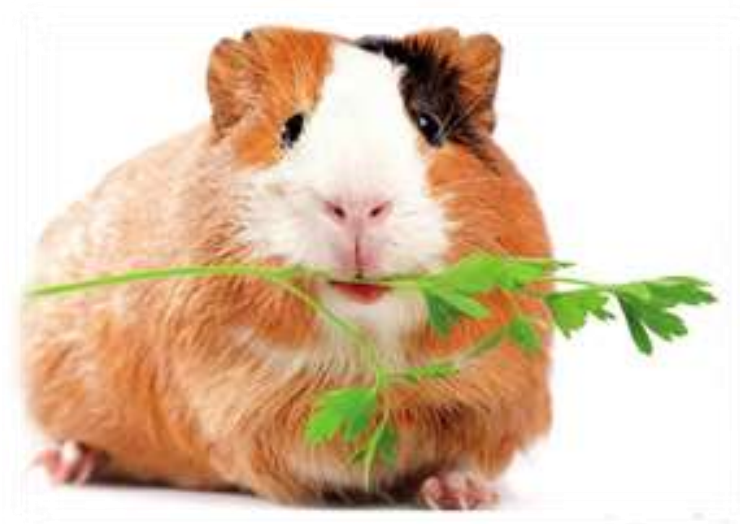

Figura 5.Cuy alimentado con Botón de oro

Fuente: Tomada de Noumbissi et al., (2013)

En un estudio realizado por Valencia et al., (2007) observaron que las aves alimentadas con diferentes niveles de Trichanthera gigantea (7, 12 y 17\%) no afectaron el promedio de GTP 2500 g, CA 1.5-2.0 en comparación con el testigo a base de concentrado comercial; sin embargo, encontraron que a mayor nivel de inclusión de $T$. gigantea se disminuyen los costos de alimentación.

Botello et al., (2011) en un estudio realizado con una mezcla de subproducto de caña enriquecido con ensilaje ácido de pescado (harina de caña proteínica) en sustitución de harina de pescado, concluyen que se puede remplazar hasta en un $14 \%$ la harina de caña proteínica en dietas para el engorde de tilapia roja sin afectar los indicadores productivos, los resultados determinaron que la GDP varió 
de $0.580 \mathrm{~g} / \mathrm{d}$ (T: $14 \%$ sustitución) a $0.597 \mathrm{~g} / \mathrm{d}$ (T: $0 \%$ sustitución). En otra especie como el caracol de tierra, Acevedo et al., (2014) encontraron que, al comparar el uso de la harina de botón de oro y matarratón para su alimentación, el testigo y el botón de oro mostraron un comportamiento similar, además registraron el mayor peso promedio inicial y final, seguido del tratamiento con incorporación de botón de oro y el tratamiento testigo (CC).

\section{CONSIDERACIONES FINALES}

La suplementación y el uso de harinas como estrategia de alimentación ha sido utilizada para satisfacer los requerimientos nutricionales de los animales de granja, debido a la baja calidad de algunos forrajes y escasez de alimentación en algunas épocas del año.

Mata et al., (2006) describen que especies arbóreas como el matarratón (Gliricidia sepium), leucaena (Leucaena leucocephala), guacimo (Guazuma ulmifolia), cayeno (Hibiscus rosa-sinensis) y morera (Morus alba) son de interés para el productor debido a su potencial de producción 30.6 y 21.93 ton/ha/ MS comestible para cayeno y morera, respectivamente; asimismo estos autores reportan que la harina de cayeno mostró una respuesta similar a la del concentrado, en relación al consumo (181.6 y $167.2 \mathrm{~g}$ ) y la ganancia de peso diaria $(81.6$ y $77.1 \mathrm{~g}$ ), dando como conclusión que este tipo de harina se constituye como una fuente energética alternativa para la alimentación de rumiantes.

Autores como Estupiñan et al., (2009) y González et al., (2006), que realizaron estudios en cerdos con harina de follaje de morera, demostraron ganancia de peso diario con buena rentabilidad sustituyendo el concentrado con harina de morena hasta en un $20 \%$, en dietas para cerdos.

\section{CONCLUSIONES}

La conservación de forrajes es una excelente alternativa de implementación que se presenta para proveer alimento en tiempo de sequía y escasez. La molienda de forrajes deshidratados es un tipo de conservación que se implementa, de acuerdo 
a la calidad nutricional, y que depende en gran parte del valor nutricional, estado fisiológico y tiempo de corte de la planta.

Al momento de elegir el tipo de forraje a implementar para elaborar harinas y su incorporación en dietas animales, se sugiere utilizar las especies nativas de la región, teniendo como exclusividad las de mejor porcentaje de proteína y demás elementos esenciales (fibra, energía, minerales), con el fin de brindar a los animales un alimento que provea los requerimiento nutricionales necesarios según la especie en producción.

También se puede resaltar que el uso de harinas derivadas de plantas forrajeras en Colombia ha demostrado excelentes resultados en rumiantes y no rumiantes, que se ve reflejado en los parámetros de producción especialmente de bovinos, cerdos y aves.

\section{REFERENCIAS BIBLIOGRÁFICAS}

1. Acevedo M, Zuluaga M. Efecto de la inclusión de diferentes tipos de harinas botón de oro (Tithonia diversifolia) y matarratón (Gliricidia sepium) en la alimentación del caracol de tierra (Helix aspersa Muller) en fase de levanteceba sobre la ganancia de peso y mortalidad. Tesis de Grado Zootecnista, Facultad de Ciencias Pecuarias, Corporación Universitaria Santa Rosa De Cabal, UNISARC. Santa Rosa de Cabal, Colombia. 2014.

2. Blass $\mathrm{C}$, Mateos $\mathrm{G}$, Rebollar P. Tablas FEDNA de composición y valor nutritivo de los alimentos para la fabricación de piensos compuestos. $3^{\text {a }}$ Ed. Fundación Española para el desarrollo de la Nutrición Animal. Madrid, España. 502 p. 2011.

3. Botello A, Viana M, Téllez E, Pullés, E, Cisneros, M, Solano G, Valdivié M, Miranda $O$, Rodríguez $Y$, Cutiño M, Savón L, Botello A. Sustitución de la harina de pescado por harina de caña proteínica para la engorda de tilapia roja. Revista Agrociencia, 45 (1): 23-31. 2011.

4. Cancio T, Roque I, Quintana M. Forraje de nacedero (Trichantera gigantea) en dietas para conejos de engorde. Revista Agrotecnia de Cuba, 34 (2): 108-114. 2010.

5. Cino D, Ruíz TE, Martínez Y, Chongo B, Díaz H. Harina de follaje de tithonia (Tithonia diversifolia) en dietas integrales para la alimentación de terneros lactantes. Resultados económicos preliminares. Revista Cubana de Ciencia Agrícola, 46 (4): 435-440. 2012.

6. Estupiñan K, Vasco D, Torres E. Evaluación de harina de forraje de Morera (Morus alba) en un sistema de levante-ceba de porcinos en confinamiento. Revista Tecnológica ESPOL-RTE, 22 (1): 81-87. 2009. 
7. Garcés A, Berrio L, Ruíz S, Serna J, Builes A. Ensilaje como fuente de alimentación para el ganado. Revista Lasallista de Investigación, 1 (1): 66-71. 2004.

8. González C, Tepper R, Ly J. Una aproximación al estudio del valor nutritivo de hojas de morera y aceite de palma en cerdos en crecimiento. Revista Científica FCV-LUZ, 16 (1): 67-71. 2006.

9. Mata MA, Sánchez DH, Cobos MA, Ortega ME, Mendoza GD, Arcos JL. Comportamiento productivo y fermentación ruminal de corderos suplementados con harina de cocoíte (Gliricidia sepium), morera (Morus alba) y tulipán (Hibiscus rosa-sinensis). Revista Científica FCV-LUZ, 16 (3): 249256. 2006.

10. Meza GA, Loor NJ, Sánchez AR, Avellaneda JH, Meza CJ, Vera DF, Cabanilla MG, Liuba GA, Meza JS, Meza FF, Ramírez MA, Moncayo OF, Cadena DL, Villamar RO, Díaz E, Rizzo LM, Rodríguez JM, López FX. Inclusión de harinas de follajes arbóreos y arbustivos tropicales (Morus alba, Erythrina poeppigiana, Tithonia diversifolia E Hibiscus rosa-sinensis) en la alimentación de cuyes (Cavia porcellus Linnaeus). Revista de la Facultad de Medicina Veterinaria y Zootecnia, 61 (3): 258-269. 2014.

11. Mejía S, Cuadrado H, Rivero T. Manejo agronómico de algunos cultivos forrajeros y técnicas para su conservación en la región Caribe Colombiana. Manual Técnico. 2ª Ed. Bogotá, Colombia, CORPOICA, 77 p. 2013.

12. Moreno F, Molina D. Manual: Buenas practicas agropecuarias -BPA- en la producción de ganado doble propósito bajo confinamiento con caña panelera como parte de la dieta. Plan de manejo sanitario del hato, condiciones de estabulación, normatividad BPA, desarrollo rural, buenas prácticas agropecuarias, seguridad alimentaria y nutricional, FAO, Gobernación de Antioquia, MANA, CORPOICA, Centro de Investigación "La Selva". 142 p. 2007.

13. Noumbissi MNB, Tendonkeng F, Zougou TG, Tedonkeng Pamo E. Efecto de diferentes niveles de suplementación con hojas de Tithonia diversifolia (Hemsl.) A. Gray sobre la ingestión y en la digestibilidad in vivo de Pennisetum purpureum K. Schum. en el conejillo de Indias (Cavia porcellus L). 32 (3):138146. 2013.

14. Parsi J, Godio L, Miazzo R, Maffioli R, Echavarria A, Provensal P. Valoración nutritiva de los alimentos y formulación de dietas. 2001. Recuperado 12 Febrero 2016. Disponible En: http://www.produccionanimal.com.ar/informacion tecnica/manejo del alimento/16valoracion nutritiva de los alimentos.pdf

15. Paziani S. Controle de perdas na ensilagem, desempenho e digestão de nutrientes em bovinos de corte alimentados com rações contendo silagens de capim tanzânia. Tesis de Doctorado, Escuela Superior de Agricultura "Luiz de Queiroz", Piracicaba, Estado de São Pablo, Brasil. 208 p. 2004.

16. Pizzani P, Blanco M, Malaver T, Godoy S, Matute I, Palma J, Obispo N. Composición fitoquímica y nutricional de harina de pijiguao (Bactris gassipaes Kunth en H.B.K). Zootecnia Tropical, 26 (3): 235-238. 2008.

17. Sarria $P$, Leterme $P$, Londoño $A$, Botero $M$. Valor nutricional de algunas forrajeras para la Alimentación de monogástricos. Asociación Venezolana de 
Producción Animal. VIII Encuentro de Nutrición y Producción en Monogástricos, Alimentación no convencional para monogástricos en el trópico. p. 115-128. 2009.

18. Savón L, Gutiérrez O, Ojeda F, Scull I. Harinas de follajes tropicales: una alternativa potencial para la alimentación de especies monogástricas. Pastos y Forrajes, 28 (1): 69-79. 2005.

19. Trómpiz J, Ventura M, Esparza D, Del Villar A, Aguirre J. Utilización de la harina de follaje de yuca (Manihot esculenta Crantz) en cerdos en crecimiento. Revista Científica FCV-LUZ. 10 (4): 315-320. 2000.

20. Trómpiz J, Ventura Max, Gómez A, Balzan M, Silva P, Barreto K. Evaluación de raciones con harina de follaje de yuca (Manihot esculenta Crantz) sobre el rendimiento productivo de cerdos en etapa de engorde. Revista Científica FCV-LUZ, 12 (2): 481-483. 2002.

21. Valencia J, Sarria E, Rivera D. Efecto de tres niveles de inclusión de nacedero (Trichanthera gigantea) y materias primas convencionales en la alimentación de pollos de engorde en el municipio de Popayán-Cauca. Trabajo de Grado Zootecnista. Escuela de Ciencias Agrícolas, Pecuarias y del Medo Ambiente, Universidad Nacional Abierta y a Distancia UNAD. Popayán, Colombia. 87 p. 2007.

22. Wagner B, Asencio V, Caridad J. Como preparar un buen ensilaje. Serie: Conservación de forrajes. Instituto Dominicano de Investigaciones Agropecuarias y Forestales (IDIAF). Santo Domingo, República Dominicana. 15 p. 2013. 